\title{
Electrothermally driven flows in ac electrowetting
}

\author{
Pablo García-Sánchez, ${ }^{1, *}$ Antonio Ramos, ${ }^{2}$ and Frieder Mugele ${ }^{1}$ \\ ${ }^{1}$ Physics of Complex Fluids, MESA + and IMPACT Institutes, Faculty of Science and Technology, University of Twente, P.O. Box 217, \\ 7500 Enschede, The Netherlands \\ ${ }^{2}$ Depto. Electrónica y Electromagnetismo, Facultad de Física, Universidad de Sevilla, Avda. Reina Mercedes s/n, 41012 Sevilla, Spain
}

(Received 16 October 2009; published 27 January 2010)

\begin{abstract}
Mixing within sessile drops can be enhanced by generating internal flow patterns using ac electrowetting. While for low ac frequencies, the flow patterns have been attributed to oscillations of the drop surface, we provide here the driving mechanism of the hitherto unexplained high-frequency flows. We show that: (1) the electric field in the liquid bulk becomes important, leading to energy dissipation due to Joule heating and a temperature increase of several degrees Celsius, and (2) the fluid flow at these frequencies is generated by electrothermal effect, i.e., gradients in temperature give rise to gradients in conductivity and permittivity, the electric field acting on these inhomogeneities induces an electrical body force that generates the flow. We solved numerically the equations for the electric, temperature and flow fields. The temperature is obtained from a convection-diffusion equation where Joule heating is introduced as a source term. From the solution of the electric field and the temperature, we compute the electrical force that acts as a body force in Stokes equations. Our numerical results agree with previous experimental observations.
\end{abstract}

DOI: 10.1103/PhysRevE.81.015303

PACS number(s): 47.65.-d, 47.61.Ne

Microfluidic flows are typically laminar [1,2]. Achieving efficient mixing is therefore a fundamental challenge in the design of microfluidic devices for (bio)chemical applications. In the absence of turbulence, chaotic flow patterns have to be generated inside the fluid leading to sufficiently thin striation layers, such that diffusive mixing can be obtained on an acceptable time scale [3]. For drop-based digital microfluidic devices such flow patterns within drops are typically generated making use of viscous shear applied to the drop via its surface. For drops in an ambient immiscible fluid phase, gradients in the viscous stresses along the drop surface control the process, for sessile drops in direct contact with a solid substrate, as in electrowetting (EW) driven devices, the no-slip boundary condition at the solid liquid interface gives rise to the typical caterpillar flow patterns if the drop is translated along the surface. Both schemes require translations over substantial distances before the liquid in the drop becomes mixed, which is inconvenient for the design of small lab-on-a-chip systems.

In electrowetting (see Ref. [4] for a general review), it was recently [5-7] reported that internal flow patterns promoting mixing can also be produced without lateral translation by using ac electric voltages, allowing for efficient "mixing on the spot." Two main regimes were identified: at ac frequencies around the typical eigenfrequency of the drop, hydrodynamic effects drive an internal flow. Upon increasing the frequency to the range of several $\mathrm{kHz}$ (for typical millimeter-sized drops) this flow gradually dies out as the liquid cannot follow the applied forcing anymore. Upon increasing the frequency to $100 \mathrm{kHz}$ and beyond, however, another very strong internal flow pattern appears, which gives rise to extremely efficient mixing [7] (see Fig. 1). The dependence on the salt concentration and on the applied ac frequency led Ko et al. [5] to the conclusion that this flow

\footnotetext{
*pablogarcia@us.es
}

must be driven by an electrokinetic mechanism that involves the penetration of electric fields into the drop. This reasoning is consistent with earlier investigations and calculations, which clearly showed a reduced electrowetting response at high frequencies due to penetration of the electric field into the drops [8-10] - in contrast to the common approach in $\mathrm{dc}$ and low-frequency EW, where the aqueous phase is considered as a perfect conductor.

To understand the qualitative behavior, let us consider the charging of the insulator by the electrical current through the liquid bulk. The insulating layer can be modeled as a capacitor in series with a resistor due to the finite conductivity of the liquid. The typical frequency for this $\mathrm{RC}$ circuit can be estimated as $(1 / 2 \pi)(\sigma d) /\left(\varepsilon_{d} L\right)$, where $\sigma$ is the liquid conductivity, $\varepsilon_{d}$ and $d$ are the dielectric constant and the thickness of the insulator, and $L$ is a typical length in the system, e.g., the distance of the needle to the electrode. The voltage drop in the liquid is negligible only for frequencies well below this characteristic frequency [8-10]. Therefore, in the case of Fig. 1(c), we can expect an important electric field and Ohmic current in the liquid that gives rise to energy dissipation. In this communication, we analyzed the effect of

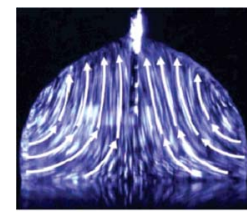

(a) $\mathrm{f}=1 \mathrm{kHz}$

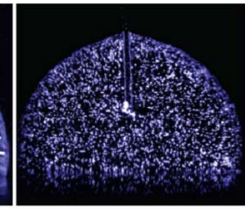

(b) $f=18 ~ k H z$

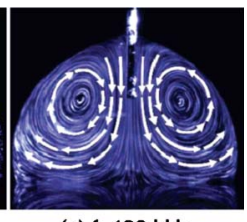

(c) $\mathrm{f}=128 \mathrm{kHz}$
FIG. 1. (Color online) Hydrodynamic flows observed in electrowetting experiments (reproduced with permission from Ref. [5]). An aqueous droplet with conductivity $1.3 \mathrm{mS} / \mathrm{m}$ and volume $5 \mu \mathrm{l}$ is subjected to an ac signal of amplitude $80 \mathrm{~V}_{\mathrm{rms}}$. (a) At low frequencies the motion is generated by the oscillation of the drop. (b) The amplitude of the oscillations decreases as the frequency increases. (c) Another type of flow appears at higher frequencies, and its origin remains unexplained. 
Joule heating in ac electrowetting experiments. The increase in temperature inside the liquid can generate flow because of natural convection, thermocapillary effect or the so-called electrothermal effect. As shown below, the heating is concentrated at the needle tip and natural convection cannot be responsible of the observations in Fig. 1(c) because buoyancy forces would produce flow rolls in the opposite direction. Furthermore, our estimation of the maximum velocity generated by the buoyancy force in the conditions of Fig. 1(c) is $0.1 \mathrm{~mm} / \mathrm{s}$, while typical velocities in experiments are around $1 \mathrm{~mm} / \mathrm{s}$. The thermocapillary effect would generate gradients in surface tension and, as a consequence, a stress tangential to the drop surface. This surface stress is directed toward lower temperatures and the flow generated by this mechanism, if any, would be again in opposite direction to the observations.

Variations in temperature within a liquid can induce gradients in its electrical properties (i.e., conductivity and permittivity). The action of an electric field on these inhomogeneities can give rise to a force in the liquid bulk and generate fluid motion. That is the meaning of electrothermally induced motion [11-13]. It has been shown that this motion can be induced in electrolytes over microelectrode structures [13-15]. Controlled heating by an external source [16], Joule heating [14], or electrodes heated by optical illumination [17] are examples of heat sources that can induce gradients in the electrical properties of the liquid.

In the remaining of this communication we will focus on the electrothermal effect in ac electrowetting. Electrical forces in the liquid bulk are given by the following expression [18]:

$$
\mathbf{f}_{E}=\rho \mathbf{E}-\frac{1}{2}|\mathbf{E}|^{2} \boldsymbol{\nabla} \varepsilon+\frac{1}{2} \boldsymbol{\nabla}\left[\rho_{m}\left(\frac{\partial \varepsilon}{\partial \rho_{m}}\right)_{T}|\mathbf{E}|^{2}\right]
$$

where $\rho$ and $\rho_{m}$ are the free electrical charge density and mass density, respectively. The last term in 1, the electrostriction, can be incorporated into the pressure for incompressible fluids [18]. The other two terms correspond, respectively, to the Coulomb and the dielectric forces.

Electrolytes are expected to be quasielectroneutral and, therefore, the Coulomb and the dielectric forces will be zero unless gradients in conductivity $\sigma$ and permittivity $\varepsilon$ are present. Let us assume that changes in temperature are small such that we can expand $\sigma$ and $\varepsilon$ around the reference temperature $T_{0}$,

$$
\sigma(T)=\sigma^{0}\left(1+\beta\left(T-T_{0}\right)\right) ; \varepsilon(T)=\varepsilon^{0}\left(1+\alpha\left(T-T_{0}\right)\right)
$$

where $\alpha=(1 / \varepsilon)(\partial \varepsilon / \partial T)$ and $\beta=(1 / \sigma)(\partial \sigma / \partial T)$ (for aqueous solution at $293 \mathrm{~K}, \alpha=-0.0046 \mathrm{~K}^{-1}$, and $\left.\beta=0.020 \mathrm{~K}^{-1}[19]\right)$. For ac signals of frequency $\omega$, we use complex amplitudes of the electric field $\mathbf{E}(t)=\operatorname{Re}(\tilde{\mathbf{E}} \exp (i \omega t))$. Then the conservation equation for the electrical charge reads as $\boldsymbol{\nabla} \cdot \mathbf{J}=\boldsymbol{\nabla} \cdot((\sigma+i \omega \varepsilon) \tilde{\mathbf{E}})=0$ (convection currents are neglected, [12]) and Gauss' law reads $\rho=\nabla \cdot(\varepsilon \widetilde{\mathbf{E}})$. Under the assumption of small changes in temperature, the electric field can be expanded to give $\widetilde{\mathbf{E}}=\widetilde{\mathbf{E}}_{0}+\widetilde{\mathbf{E}}_{1},\left(\left|\widetilde{\mathbf{E}}_{1}\right| \ll\left|\widetilde{\mathbf{E}}_{0}\right|\right.$, where $\widetilde{\mathbf{E}}_{0}$ is the electric field for an homogeneous temperature $T_{0}$ ), where the electric field satisfies

$$
\begin{gathered}
\boldsymbol{\nabla} \cdot \tilde{\mathbf{E}}_{0}=0 \\
\nabla \cdot \tilde{\mathbf{E}}_{1}+\left(\frac{\boldsymbol{\nabla} \sigma+i \omega \boldsymbol{\nabla} \varepsilon}{\sigma^{0}+i \omega \varepsilon^{0}}\right) \cdot \widetilde{\mathbf{E}}_{0}=0
\end{gathered}
$$

and the zeroth-order electric field can be derived from an electrical potential which satisfies Laplace's equation $\left(\widetilde{\mathbf{E}}_{0}=-\nabla \Phi, \nabla^{2} \Phi=0\right)$. The charge density of each order corresponds to

$$
\begin{gathered}
\rho_{0}=\varepsilon^{0} \boldsymbol{\nabla} \cdot \widetilde{\mathbf{E}}_{0}=0 \\
\rho_{1}=\varepsilon^{0} \boldsymbol{\nabla} \cdot \widetilde{\mathbf{E}}_{1}+\nabla \varepsilon \cdot \tilde{\mathbf{E}}_{0}=\left(\frac{\sigma^{0} \nabla \varepsilon-\varepsilon^{0} \nabla \sigma}{\sigma^{0}+i \omega \varepsilon^{0}}\right) \cdot \tilde{\mathbf{E}}_{0}
\end{gathered}
$$

The time-averaged electrical force can then be written as [12]

$$
\left\langle\mathbf{f}_{E}\right\rangle=\frac{1}{2} \operatorname{Re}\left[\frac{\varepsilon^{0}(\alpha-\beta)}{1+i \omega \tau}\left(\nabla T \cdot \widetilde{\mathbf{E}}_{0}\right) \widetilde{\mathbf{E}}_{0}^{*}-\frac{1}{2} \varepsilon^{0} \alpha\left|\widetilde{\mathbf{E}}_{0}\right|^{2} \boldsymbol{\nabla} T\right]
$$

where $\tau \equiv \varepsilon^{0} / \sigma^{0}$ is the charge relaxation time of the liquid and $*$ indicates the complex conjugate. Expression (7) is frequency dependent. For $\omega$ below the charge relaxation frequency of the liquid $\left(\sigma^{0} / \varepsilon^{0}\right)$ the Coulomb force dominates because relative variations in conductivity $(\nabla \sigma / \sigma)$ are usually much greater than relative variations in permittivity $(\nabla \varepsilon / \varepsilon)$. The relaxation frequency for typical conductivities in ac electrowetting $\left(10^{-3}\right.$ to $\left.1 \mathrm{~S} / \mathrm{m}\right)$ is much larger than the frequencies applied in experiments and, therefore, we will only consider the Coulomb forces in our calculations.

The expression for the force contains both a timeaveraged dc component and an oscillatory component of frequency $2 \omega$ since both $\rho$ and $\mathbf{E}$ oscillate with $\omega$. The latter gives rise to a small amplitude rapid oscillation of the liquid, which is impossible to observe in practice. We are interested in the time-averaged component of the velocity, which is computed from the Navier-Stokes equation in the low Reynolds number approximation together with the mass conservation equation for an incompressible fluid,

$$
\begin{gathered}
\eta \nabla^{2} \mathbf{u}-\nabla p+\mathbf{f}=0 \\
\boldsymbol{\nabla} \cdot \mathbf{u}=0
\end{gathered}
$$

where $p$ is the pressure, $\eta$ is the viscosity of the fluid, $\mathbf{f}$ is the body force applied on the liquid. In this case, the body force is the Coulomb force term in Eq. (7). As is usually done in the Boussinesq approximation [20], we neglect variations of liquid viscosity with temperature.

For sufficiently high frequencies [12], the temperature field can be obtained from the steady-state energy balance equation

$$
\rho_{m} c_{p} \mathbf{u} \cdot \nabla T=k \nabla^{2} T+(1 / 2) \sigma^{0}\left|\mathbf{E}_{0}\right|^{2}
$$

where $c_{p}$ is the specific heat at constant pressure and $k$ is the thermal conductivity. The last term corresponds to the timeaveraged energy dissipation by the electric current at zeroth order.

We use COMSOL, a commercial software implementing the 


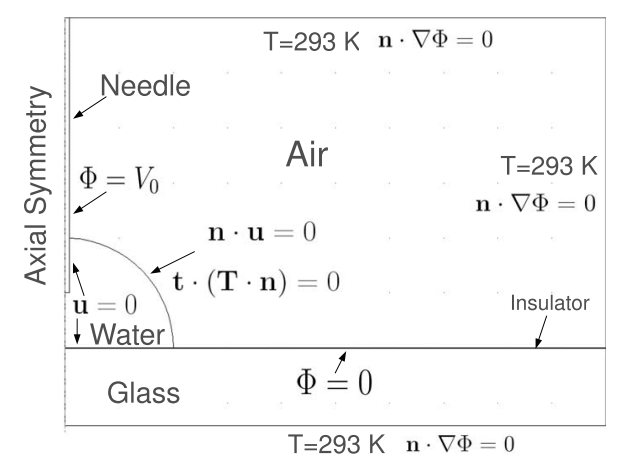

FIG. 2. Problem domain. The problem has axial symmetry and a semispherical cap is assumed for the drop shape. A domain much larger than the drop is considered so at the outer boundaries we impose room temperature and zero electrical current.

finite element method, for solving the equations above. We take advantage of the axial symmetry of the problem and solve the equations in a domain like the one in Fig. 2. For simplicity, we assume that the drop has a constant semispherical cap shape. We will show that the fluid flow is generated at the needle and, therefore, changes in the value of the contact angle are not relevant for the general behavior of the problem. All geometrical dimensions and material properties are chosen according to the experiments by Ko et al. (see also [8]), drop and needle sizes, insulator thickness, and dielectric constants and thermal conductivities of all materials. The equation for the temperature is solved in the whole domain, assuming room temperature $(T=293 \mathrm{~K})$ at the outer boundaries and continuity of the heat flux in all interior boundaries. The heat convection in the air is neglected, which is a good approximation in our problem. In effect, the value of the Peclet number for heat transport in air with typical velocity $1 \mathrm{~mm} / \mathrm{s}$ and length $1 \mathrm{~mm}$ is $\mathrm{Pe} \sim 0.045$. The equation for the electric field is solved everywhere but in the needle, which is considered a perfect conductor at the potential of source $\Phi=V_{0}$. At all outer boundaries we impose the condition of zero electrical current $\mathbf{n} \cdot \nabla \Phi=0$ and the electrode on top of the glass is at zero potential $\Phi=0$. At all other interior boundaries we impose continuity of the complex electrical current. Equations (8) are solved within the drop, imposing no-slip on the needle and on the insulator. Because of the very low dynamic viscosity of air, we con-
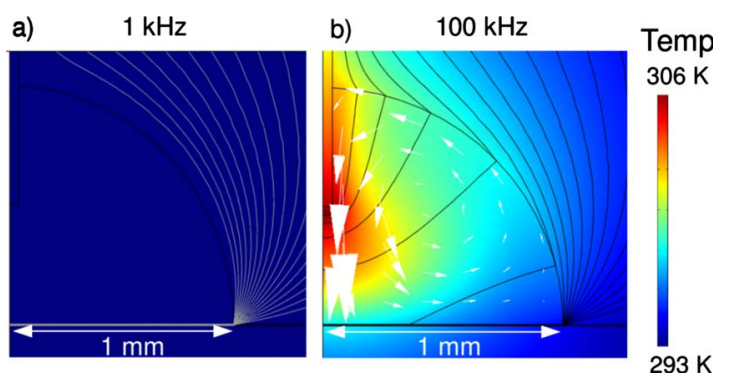

FIG. 3. (Color online) Solution for the electric potential (contour lines), temperature (surface plot) and velocity field (arrows) for a conductivity of $1.3 \times 10^{-2} \mathrm{~S} / \mathrm{m}$ and a signal of $100 \mathrm{~V}$ and two different frequencies [(a)1 kHz and (b) $100 \mathrm{kHz}$. Typical velocities in (b) are around $1 \mathrm{~mm} / \mathrm{s}$.

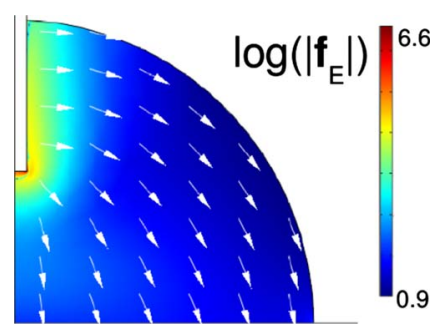

FIG. 4. (Color online) Electrothermal force for the case of Fig. 3 (b), $\left(1.3 \times 10^{-2} \mathrm{~S} / \mathrm{m} 100 \mathrm{~V}, 100 \mathrm{kHz}\right)$. Normalized arrows indicate the force direction. The force is only intense near the tip of the needle and with a maximum magnitude of nearly $4 \times 10^{6} \mathrm{~N} / \mathrm{m}^{3}$.

sider the water-air interface as a free surface-zero shear stress- $(\mathbf{n} \cdot \mathbf{u}=0$ and $\mathbf{t} \cdot(\mathbf{T} \cdot \mathbf{n})=0$, where $\mathbf{T}$ is the stress tensor, $\left.\mathbf{T}=-p \mathbf{I}+\eta\left(\boldsymbol{\nabla} \mathbf{u}+\nabla \mathbf{u}^{T}\right)\right)$.

Figure 3 is a plot of the solution to the temperature field (surface plot), voltage (contour lines) and flow field (arrows) for a conductivity of $1.3 \times 10^{-2} \mathrm{~S} / \mathrm{m}$ and a signal of $100 \mathrm{~V}$ at two different frequencies. At $1 \mathrm{kHz}$ the temperature is constant in the domain (no Joule heating), the voltage within the drop is constant, and there is no fluid flow. However, at $100 \mathrm{kHz}$ the voltage is not constant within the drop and the resultant electrical current leads to an increase in temperature. Also, there appears a fluid flow as a consequence of the electrothermal effect. The flow pattern is like the one in Fig. 1 (c) and typical velocities are around $1 \mathrm{~mm} / \mathrm{s}$. This is in qualitative agreement with the results of Ref. [5] from which a maximum flow speed of at most a few $\mathrm{mm} / \mathrm{s}$ can be inferred.

In Fig. 4 we plot the electrothermal force for the case of Fig. 3(b). Normalized arrows indicate the direction of the force. The force is very intense near the tip of the needle (pointing downwards) and rapidly becomes negligible. This can be observed in the surface plot of the logarithm of the force magnitude. This result is also in agreement with the observation in Ref. [5] where the position of the needle was changed and it was observed that the position of the flow rolls changed accordingly.

Figure 5 shows the dependence of the velocity on the frequency for a voltage amplitude of $50 \mathrm{~V}$. The velocity values are taken at a position near the needle $(600 \mu \mathrm{m}$ above the insulator and $60 \mu \mathrm{m}$ from the symmetry axes). For

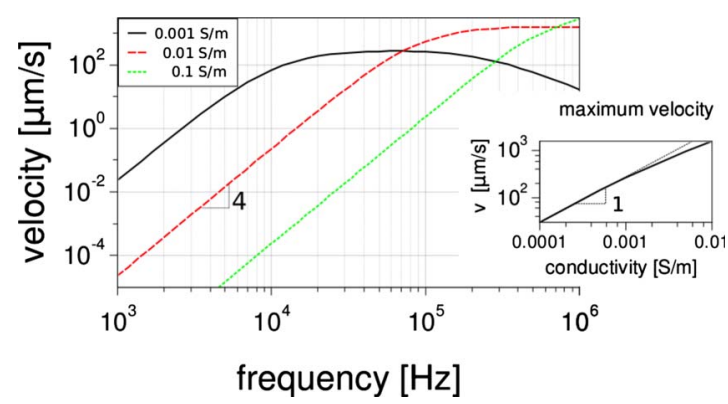

FIG. 5. (Color online) Fluid velocity generated by electrothermal effect versus frequency of the applied signal. The amplitude of the signal is $50 \mathrm{~V}$. The inset shows the maximum velocity obtained for each conductivity. 
$\sigma=0.001 \mathrm{~S} / \mathrm{m}$ and $\sigma=0.01 \mathrm{~S} / \mathrm{m}$, the velocity reaches a maximum around $60 \mathrm{kHz}$ and $600 \mathrm{kHz}$, respectively, which corresponds to the relaxation frequency of the equivalent $\mathrm{RC}$ circuit for each conductivity. For $\sigma=0.1 \mathrm{~S} / \mathrm{m}$, the maximum velocity would be found around $6 \mathrm{MHz}$. We have included an inset with a plot of the maximum velocity obtained for each conductivity. For the lowest conductivities, the velocity increases linearly with the conductivity, as expected in the case of gradients of temperature generated by Joule heating $(\nabla T \sim \sigma)$. At higher conductivities and, consequently, higher velocities this scaling is not true because the convection term in Eq. (10) becomes important and the gradient in temperature is smoothed. Figure 5 shows how the motion rapidly vanishes when the frequency decreases and higher frequencies are required if the conductivity increases.

Figure 6 is a plot of the velocity versus the amplitude of the applied signal for a constant frequency of $50 \mathrm{kHz}$. The velocity scales with the fourth power of the voltage amplitude, as expected from the scaling of the force [Eq. (7)] since the gradients of temperature scale with the second power of voltage $\left(\nabla T \sim V^{2}\right)$. This is not true, however, at the highest velocities in Fig. 6. Again, the convection term becomes important and the gradients in temperature are smoothed.

In summary, we analyzed the effect of Joule heating in electrowetting experiments with ac signals. For typical amplitudes and frequencies, the increase in temperature can be important and have dramatic consequences when working with biological samples. We computed the flow field generated by electrothermal effect and the results agree with previous experimental observations of fluid flow in $\mathrm{AC}$ electrowetting.

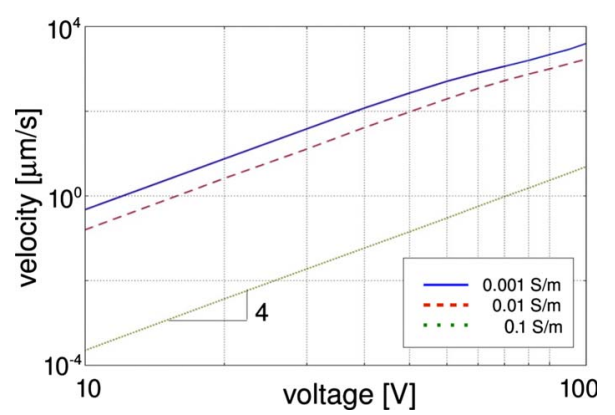

FIG. 6. (Color online) Fluid velocity generated by electrothermal effect versus amplitude of the applied signal. The frequency of the signal is $50 \mathrm{kHz}$.

The analysis here presented can be extended to other electrode structures in electrowetting applications. From an applied perspective, it will be crucial to minimize the heating required to drive efficient mixing flows, e.g., by optimizing electrode geometries, in order to avoid interference with potentially temperature sensitive biochemical subtances.

Note Added. During the reviewing process of this Rapid Communication, we became aware that a very similar work was carried out in parallel by Kang et al. (submitted to Biomicrofluidics) and reached exactly the same conclusions regarding the driving force of the high-frequency internal flows [21].

P.G.-S. acknowledges MicroNed, the Microtechnology Research Programme of The Netherlands, for financial support.
[1] H. Stone, A. Stroock, and A. Ajdari, Annu. Rev. Fluid Mech. 36, 381 (2004).

[2] T. M. Squires and S. R. Quake, Rev. Mod. Phys. 77, 977 (2005).

[3] J. M. Ottino and S. Wiggins, Philos. Trans. R. Soc. London, Ser. A 362, 923 (2004).

[4] F. Mugele and J.-C. Baret, J. Phys.: Condens. Matter 17, R705 (2005).

[5] S. H. Ko, H. Lee, and K. H. Kang, Langmuir 24, 1094 (2008).

[6] F. Mugele, J.-C. Baret, and D. Steinhauser, Appl. Phys. Lett. 88, 204106 (2006).

[7] K. P. Nichols and H. J. Gardeniers, Anal. Chem. 79, 8699 (2007).

[8] J. S. Hong, S. H. Ko, K. H. Kang, and I. S. Kang, Microfluid. Nanofluid. 5, 263 (2008).

[9] T. B. Jones, Langmuir 18, 4437 (2002).

[10] A. Kumar, M. Pluntke, B. Cross, J. C. Baret, and F. Mugele, Finite Conductivity Effects and Apparent Contact Angle Saturation in AC Electrowetting, MRS Symposia Proceedings Vol. 899 (Materials Research Society, Warrendale, PA, 2005), pp. 69-76.

[11] J. R. Melcher and M. S. Firebaugh, Phys. Fluids 10, 1178
(1967).

[12] A. Ramos, H. Morgan, N. Green, and A. Castellanos, J. Phys. D 31, 2338 (1998).

[13] A. González, A. Ramos, H. Morgan, N. G. Green, and A. Castellanos, J. Fluid Mech. 564, 415 (2006).

[14] G. Fuhr, R. Hagedorn, T. Muller, W. Benecke, and B. Wagner, J. Microelectromech. Syst. 1, 141 (1992).

[15] N. G. Green, A. Ramos, A. González, A. Castellanos, and H. Morgan, J. Electrost. 53, 71 (2001).

[16] M. Felten, P. Geggier, M. Jäger, and C. Duschl, Phys. Fluids 18, 051707 (2006).

[17] N. G. Green, A. Ramos, A. González, A. Castellanos, and H. Morgan, J. Phys. D 33, L13 (2000).

[18] J. A. Stratton, Electromagnetic Theory (McGraw-Hill, New York, 1941).

[19] CRC Handbook of Chemistry and Physics, edited by D. Lide (CRC Press, New York, 1994).

[20] Electrohydrodynamics, edited by A. Castellanos (Springer, New York, 1998).

[21] H. Lee, S. Yun, S. K. Ko, and K. H. Kang, Biomicrofluidics 3, 044113 (2009). 